\title{
SWI/SNF Complex Subunit SMARCC1
}

National Cancer Institute

\section{Source}

National Cancer Institute. SWIISN F Complex Subunit SMARCC1. NCI Thesaurus. Code C19838.

SWI/SNF complex subunit SMARCC1 (1105 aa, 123 kDa) is encoded by the human SMARCC1 gene. This protein plays a role in both chromatin remodeling and transcriptional regulation. 